\title{
Desenvolvimento de objetos de aprendizagem modernos em Teleodontologia
}

\author{
Cássio José Fornazari Alencar*, Érika Sequeira**, Chao Lung Wen***, Ana Estela
} Haddad****

\author{
* Doutor em Odontopediatria - Faculdade de Odontologia da \\ Universidade de São Paulo, Professor de Odontopediatria \\ UNIP-Campinas, Professor de Cirurgia em Odontopediatria - \\ FUNDECTO/FOUSP \\ ** Doutora em Telemedicina - Faculdade de Medicina da \\ Universidade de São Paulo \\ *** Professor Associado do Departamento de Patologia - Telemedicina, \\ Faculdade de Medicina da Universidade de São Paulo \\ ***** Professora Livre Docente Associada do Departamento de \\ Ortodontia e Odontopediatria, Faculdade de Odontologia da \\ Universidade de São Paulo
}

\section{RESUMO}

Este trabalho tem objetivo de mostrar o Homem Virtual como objeto de aprendizagem e suas etapas de confecção. Considerando a necessidade de se modernizar as iconografias e difundir conhecimentos, foi elaborado ferramentas que permitam transmitir informações de forma mais eficiente e interativa. Através do Projeto Homem Virtual da Disciplina de Telemedicina FMUSP em parceria com a FOUSP foi desenvolvido imagens de alta qualidade visual e didática. Através deste objeto de aprendizagem assiste-se a imagens de estruturas anatômicas em 3D com movimentos fisiológicos, observa-se à biomecânica e a dinâmica funcional que seriam impossíveis de serem demonstradas por métodos convencionais. A possibilidade de estabelecer correlações anatômicas, de aplicar recursos de transparências, subtração (exclusão) e inclusão de dinâmica funcional, o torna uma iconografia inédita para transmissão de grandes quantidades de informações em curto espaço de tempo, aumentando a eficiência educacional. As imagens são usadas para apoiar o estudo dos alunos e a capacitação de profissionais em qualquer local de ensino superior odontológico. Através deste projeto, há grande benefício em transmitir o conhecimento de horas de aula teórica em minutos de estudo dirigido, o que significa um aspecto inovador e motivador. Este recurso educacional, como objeto de aprendizagem, propor- ciona a integração dos conhecimentos, desperta a curiosidade e aumenta a velocidade do aprendizado fazendo parte de uma estrutura cognitiva moderna, onde se participa de forma consciente como sujeito do processo.

\section{DESCRITORES}

Telemedicina. Educação em odontologia. Educação à distância. Anatomia.

\section{INTRODUÇÃO E REVISÃo}

Diante do pequeno número de produção científica em educação mediada por tecnologia em odontologia, atualmente, este modelo educacional tem sido mais estudado em outras áreas. Os grandes centros de pesquisa destinados ao aprimoramento tecnológico se preocupado com a educação a distância (EAD) e/ou teleducação interativa, vem encontrando ambiente propício para o seu crescimento e difusão devido ao desenvolvimento das redes digitais de comunicação e a ampliação do uso da Internet. Hoje, falar desse tema implica considerar, de forma indissociável, o papel do computador e das redes digitais de transmissão da informação (Côrrea, 2001).

A Teleducação Interativa é um modelo de educação apoiado em tecnologia, que utiliza recursos de computação e interação online para promover uma capacitação dos estudantes em nível cognitivo, de 
raciocínio, desenvolvimento do comportamento frente a situações críticas, da capacidade associativa e das habilidades práticas. Nesse contexto, o qual inclui necessariamente a produção de material didático eletrônico, a Internet assume um destaque como meio pelo qual as informações são disponibilizadas e acessadas. Particularmente as linguagens HTML e XML, veiculadas pela Internet (Web) e que permitem produção de hipertextos, proporcionam mecanismos de acesso às informações que podem favorecer o auto-aprendizado. Por serem recursos tecnológicos, todo o potencial ainda tem muito a ser discutido como ferramenta para oferecer suporte aos modelos pedagógicos.

A área da Educação vem buscando, juntamente com a da Informática, propor melhoria no processo educacional, através do uso de ferramentas digitais. Uma das pesquisas mais atuais para criação dessas ferramentas, relaciona-se aos objetos de aprendizagem, que visam proporcionar uma maior interatividade na forma de transmissão de conteúdos (Machado e Silva, 2005).

Um objeto de aprendizagem tem como função atuar como recurso didático interativo, abrangendo um determinado segmento de uma disciplina e agrupando diversos tipos de dados como imagens, textos, áudios, vídeos, exercícios, e tudo o que pode auxiliar o processo de aprendizagem. Pode ser utilizado tanto no ambiente de aula, quanto no ambiente virtual - como complemento, revisão ou reforço de um determinado conteúdo já estudado. Como afirmam Bettio e Martins (2004), eles

\footnotetext{
"vêm para facilitar e melhorar a qualidade do ensino, proporcionando aos tutores, alunos e administradores, diversas ferramentas facilitadoras".
}

Na Odontologia, a educação a distância tem merecido atenção de algumas universidades americanas, inglesas e australianas, também com poucos resultados disponíveis na literatura científica. Dentre esses trabalhos, encontram-se pesquisas envolvendo a produção de material didático e o uso da Internet no ensino de Odontologia, com uma abordagem mais "empírica" do processo:

- cria-se o CDROM a partir da conversão de documentos analógicos para digitais, mas são poucos os trabalhos que verificam a sua aplicação;

- disponibiliza-se na Internet o material produzido, mas pouco se avalia o seu valor didático;

- enfim, os estudos estão em fases iniciais e ainda precisam ser melhor explorados metodologicamente.

Já na Medicina, as grandes distâncias entre os centros médicos especializados e os locais mais carentes, além de recursos escassos e mal distribuídos, representam dificuldades na área da saúde que podem ser diminuídas com a telemedicina (Maceratini, Sabbatini, 1994). Embora já existam outras definições (Chao LW, 2006), a Telemedicina também é definida como a oferta de serviços ligados aos cuidados com a saúde, nos casos em que a distância é um fator crítico. Tais serviços são oferecidos por profissionais da área de saúde, usando tecnologias de informação e de comunicação, visando o intercâmbio de informações válidas para pesquisas, diagnósticos, prevenção e tratamento de doenças e contínua educação de provedores de cuidados com a saúde, no interesse de melhorar a saúde das pessoas e de suas comunidades (OMS). Praticamente quase todas as especialidades médicas e demais áreas da saúde podem vir a utilizar-se da telemedicina, ou como adotado pelo Ministério da Saúde, telessaúde, particularmente aquelas que utilizam imagens como meio diagnóstico. Assim, os setores de radiologia, dermatologia, patologia, ultra-sonografia, oftalmologia, entre outros, são bastante propícios para o estabelecimento de protocolos de transmissão de dados à distância, com finalidades diagnósticas (Chao et al., 2003).

Trazendo este conceito e as ferramentas da Telemedicina, para a Odontologia, temos a Teleodontologia; abordando tanto os aspectos da teleducação interativa, como da teleassistência (Chao LW, 2003). Dentre estas ferramentas da Telemedicina, criada pela equipe da Faculdade de Medicina da Univesidadede São Paulo, pode-se citar:

- o Cyberambulatório,

- o Cybertutor e

- o Projeto Homem Virtual (Chao et al., 2003).

O Projeto Homem Virtual representa um novo método de comunicação dinâmica e dirigida, sendo considerado um objeto de aprendizagem moderno (www.projetohomemvirtual.com.br).

O Projeto Homem Virtual é a representação gráfica de grande número de informações especializadas, de forma interativa, dinâmica e objetiva. Usando tecnologia de modelagem gráfica em 3D, o Homem Virtual é uma forma eficiente de transmitir conhecimentos de anatomia, fisiologia, fisiopatologia e me- 
canismos moleculares. Representa uma efetiva modernização nas iconografias educacionais, uma vez que facilita e agiliza o entendimento em relação a um assunto específico. Pode ser utilizada em todos os níveis educacionais. Na Odontologia foram desenvolvidos dois temas:

- Articulação têmporo-mandibular (Figura 1) e

- Estrutura Dental (Figura 2)

E já estão em desenvolvimento:

- Desordens têmporo-mandibulares (Figura 3) e

- Anestesia/Exodontia (Figuras 4 e 5).

Essas ferramentas possibilitam que a educação a distância e a educação continuada seja realizada com objetivos concretos, fundamentados e de forma atualizada e segura. Outras características dos objetos de aprendizagem modernos (Machado e Silva, 2005;

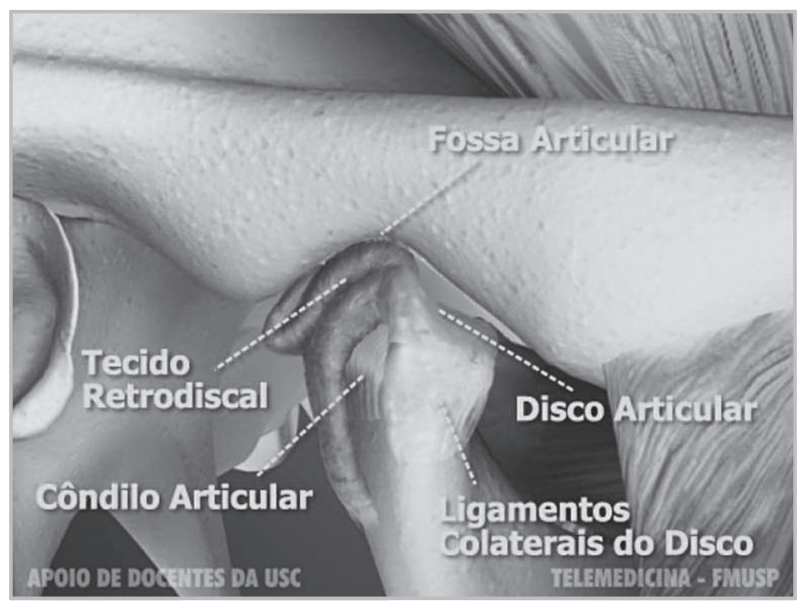

Figura 1 - Estruturas da ATM.
Bettio e Martins, 2004) são:

- flexibilidade: caracteriza-se pela permissão da criação de novos cursos utilizando-se conhecimentos já escritos e consolidados. Isso garante que o uso da tecnologia tenha uma maior credibilidade, pois eles se sustentam por fontes seguras de informação;

- customização: possibilidade de que cada entidade educacional os utilize e arranje da maneira que melhor lhe convier e de que as pessoas possam montar seus próprios conteúdos programáticos, agrupando os módulos conforme seu interesse (on-demand learning);

- interoperabilidade: consiste na utilização e reutilização dos objetos de aprendizagem em qualquer plataforma de ensino em todo o mundo, pois eles serão desenvolvidos de tal forma que possam ser utilizados em diversos sistemas;

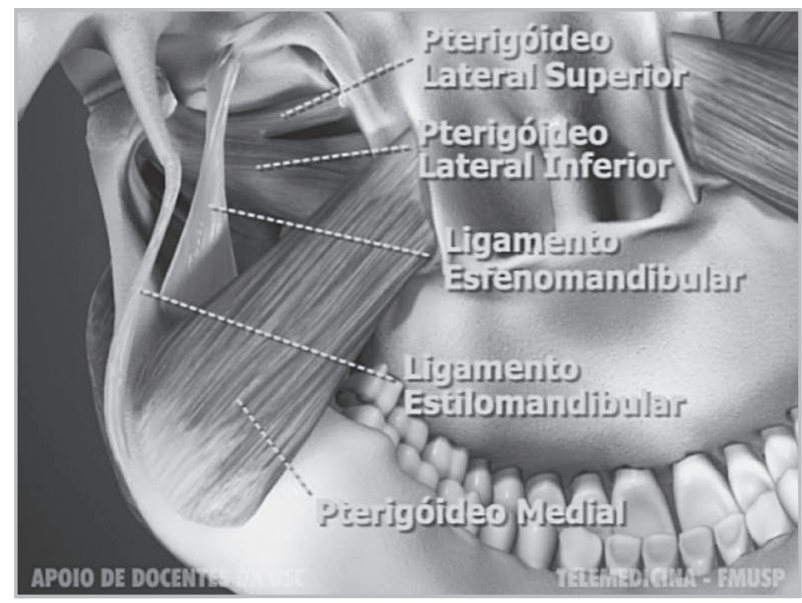

Figura 3 - Musculatura relacionada a DTM.

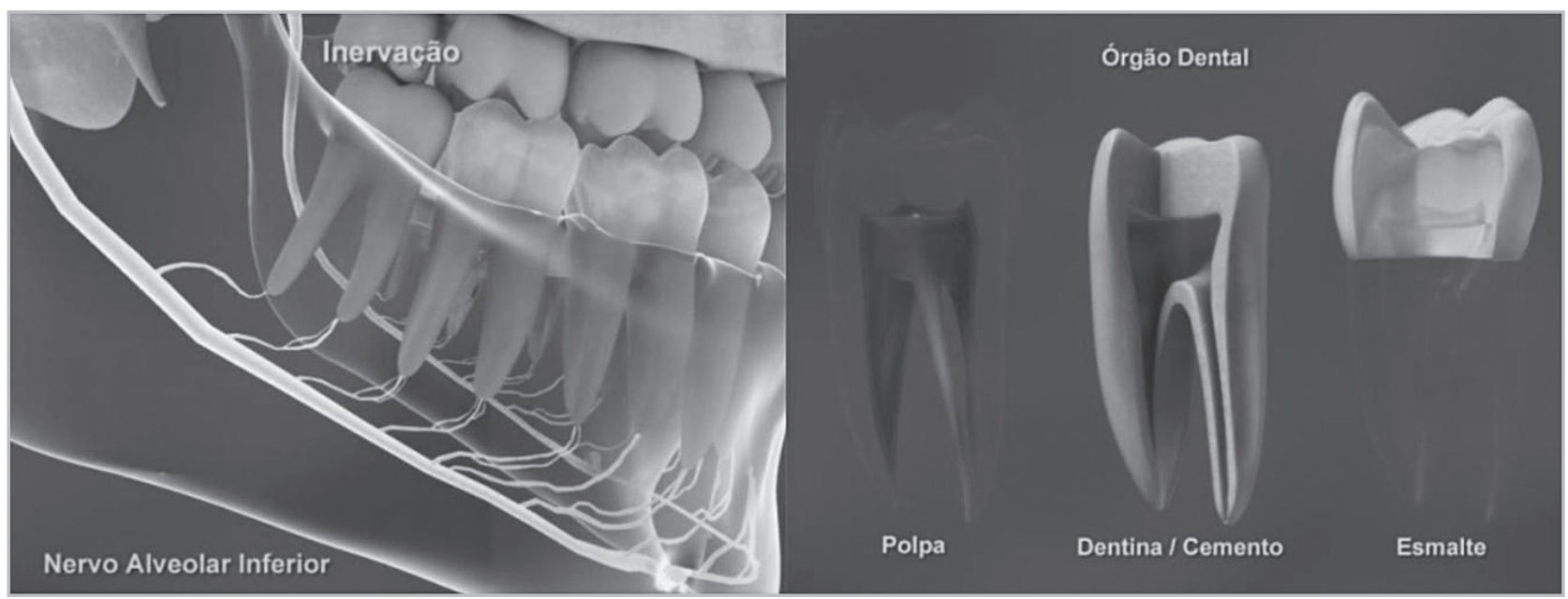

Figura 2 - Estrutura dental. 
- indexação e procura: caracteriza a padronização dos objetos de aprendizagem e dos locais onde eles são armazenados, para que a localização de um conteúdo se torne mais viável (Figura 6).

É urgente a modernização educacional da odontologia diante do atual cenário tecnológico e da realidade nacional de saúde. Diversas necessidades surgirão em breve em decorrência da atual política de educação na saúde do Ministério da Saúde, tal como o Programa Nacional de Telessaúde em Apoio à Atenção Básica, que exigirá um componente formativo odontológico para o treinamento das equipes de Saúde da Família. O Programa tem como objetivo integrar em rede as equipes da Estratégia de Saúde da Família em todas as regiões do país, especialmente nas regiões mais remotas, com Núcleos de Telessaúde vinculados a universidades, e com a Rede Universitária de Telemedicina, oferecendo às equipes apoio ao diagnóstico e tratamento, e teleducação, conforme a demanda, contribuindo para a qualidade e resolubilidade da atenção à saúde prestada à população. Vale ressaltar que das 27 mil equipes distribuídas atualmente por todo o território nacional, mais da metade conta com dentistas (Haddad et al., 2007). O presente trabalho propõe-se a demonstrar as etapas de desenvolvimento do objeto de aprendizagem - projeto Homem Virtual - em Odontologia.

\section{METODOLOGIA - ETAPAS DE CONSTRUÇÃO}

O desenvolvimento deste modelo educacional requer a aliança de conhecimentos na área de odontologia, tecnologia da informação, telemedicina e teleducação interativa.

A estruturação do plano tecnológico e estratégias de teleducação interativa, serão feitos com o apoio da Disciplina de Telemedicina da FMUSP sob a coorde-

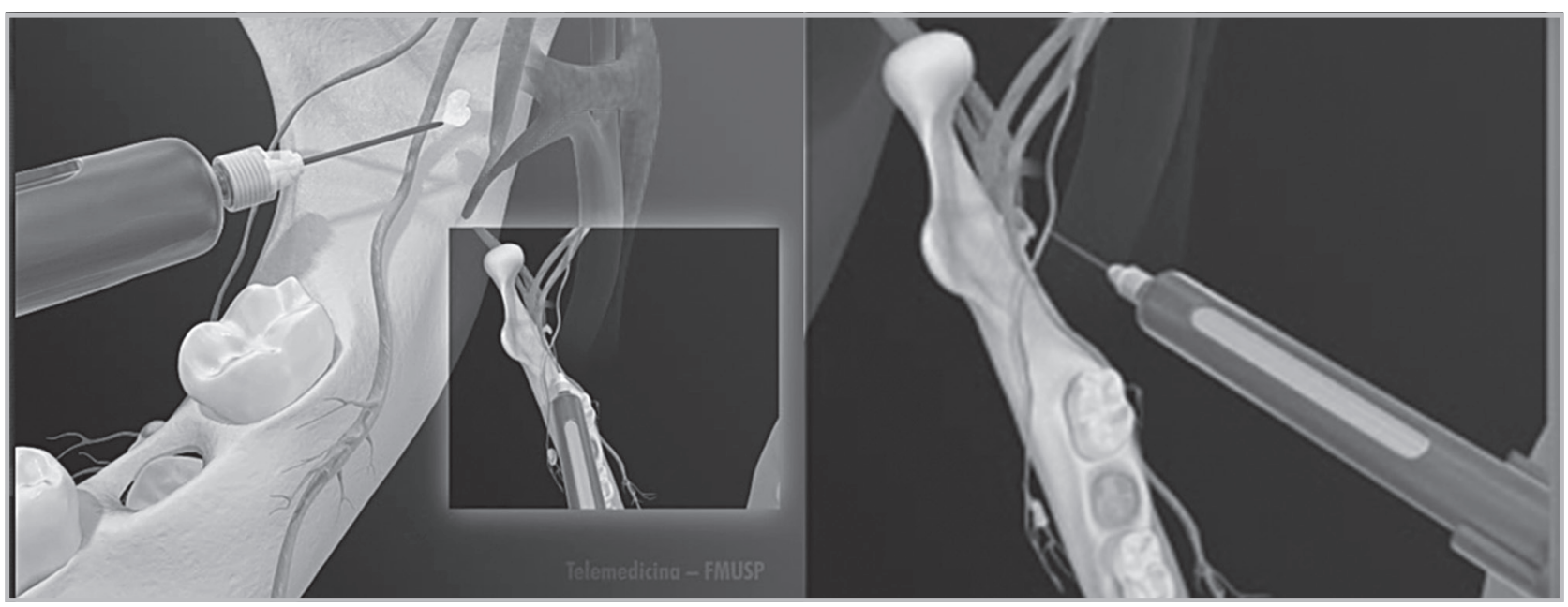

Figura 4 - Técnica Anestésica.

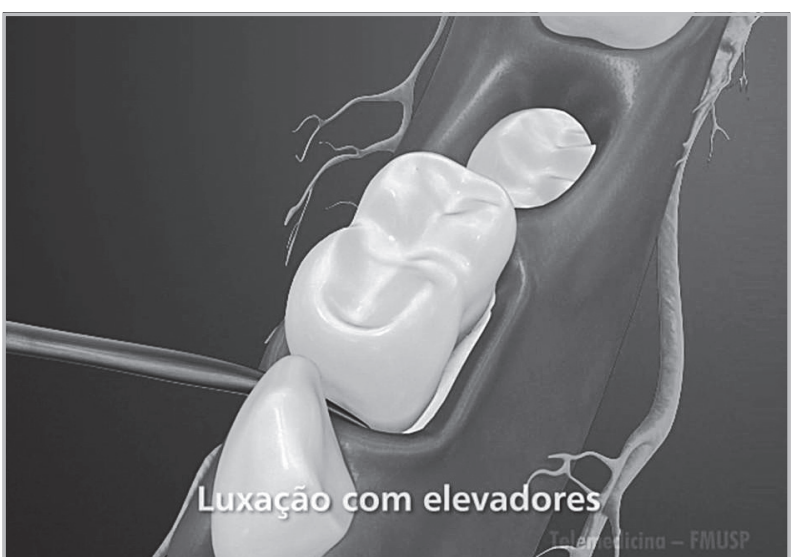

Figura 5 - Técnica de exodontia.

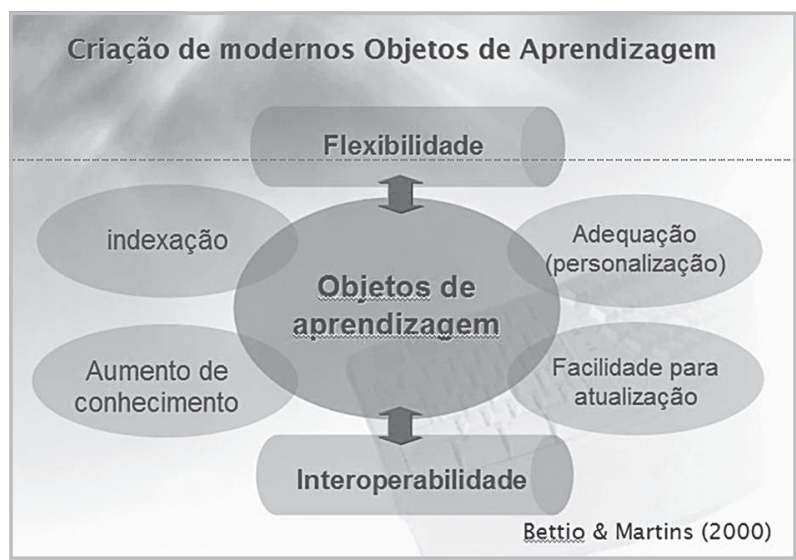

Figura 6 - Características dos objetos de aprendizagem. 
nação do co-orientador.

\section{Equipe multiprofissional}

- Especialista no assunto (profissional).

- Estrategista de Telemedicina (educador).

- Digital designers.

\section{Etapas de desenvolvimento}

- Definição da temática e dos objetivos: Estruturar o tema e as estratégias necessárias para que o objeto de aprendizagem tenha fundamento no processo ensino-aprendizagem.

- Avaliação da abrangência e público-alvo: Estudar o público-alvo e verificar a forma de abordagem para este público.

- Levantamento de literatura científica: Saber se já não existe o mesmo tema desenvolvido, ou o que se têm na literatura sobre o tema escolhido.

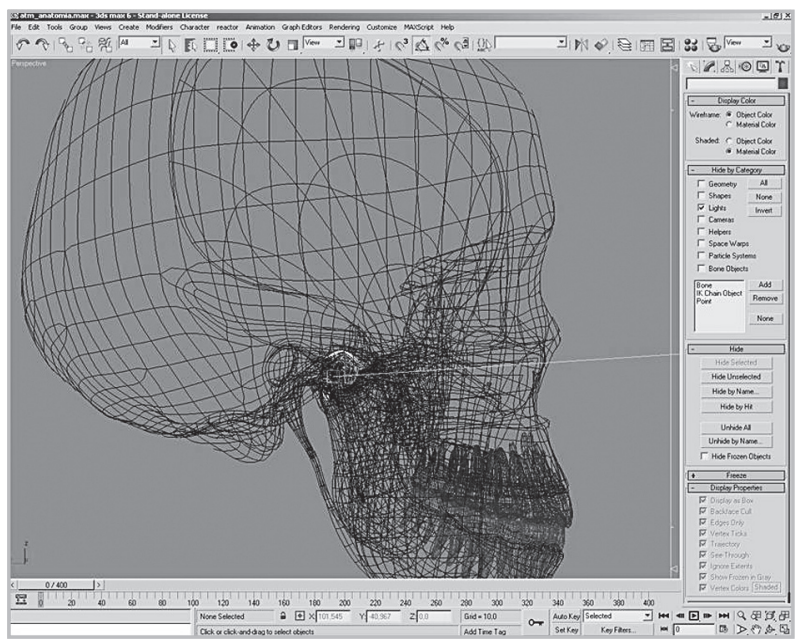

Figura 7 - Modelagem.

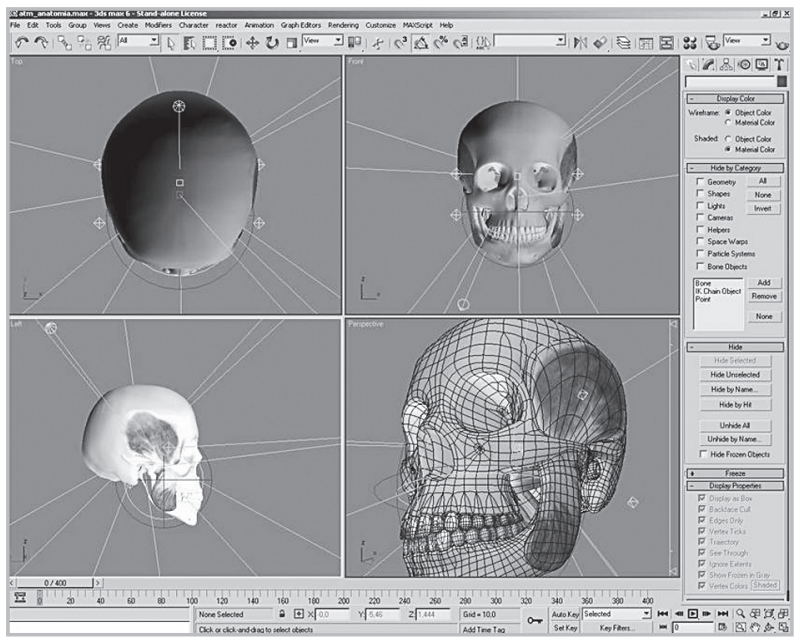

Figura 8 - Pré-visualização.
- Elaboração de estratégia de roteiro educacional: Trabalhar no conteúdo que vai ser apresentado, traçando início, meio e fim.

- Modelagem gráfica computacional (Figura 7): Utilização de softwares para confecção de estruturas.

- Geração da Pré-visualização (Figura 8): Análise de câmeras, tomadas, ângulo de visão e preview do trabalho realizado.

- Revisão científica: Rever o conteúdo formatado dentro dos conceitos e embasados na literatura científica.

- Implementação de legendas e geração do formato texturizado e renderizado (Figura 9): Finalização do trabalho com conteúdos complementares (som, escritos).

- Acompanhamento do impacto educacional: Análise de impacto e eficiência como material de aprendizagem.

\section{RECURSOS NECESSÁRIOS Infra-estrutura tecnológica/profissional:}

- Equipe do Projeto Homem Virtual - Digital Designers.

- 4 Estações Gráficas:

- Dual Pentiun IV Xeon HT;

- 4 Gigabytes de memória RAM;

- placa de vídeo profissional;

- tablet;

- programa 3D Studio Max;

- programa Photoshop ou equivalente e programa After effects.

- Equipe de desenvolvimento de website e ambiente educacional.

- 7 Estações de trabalho:

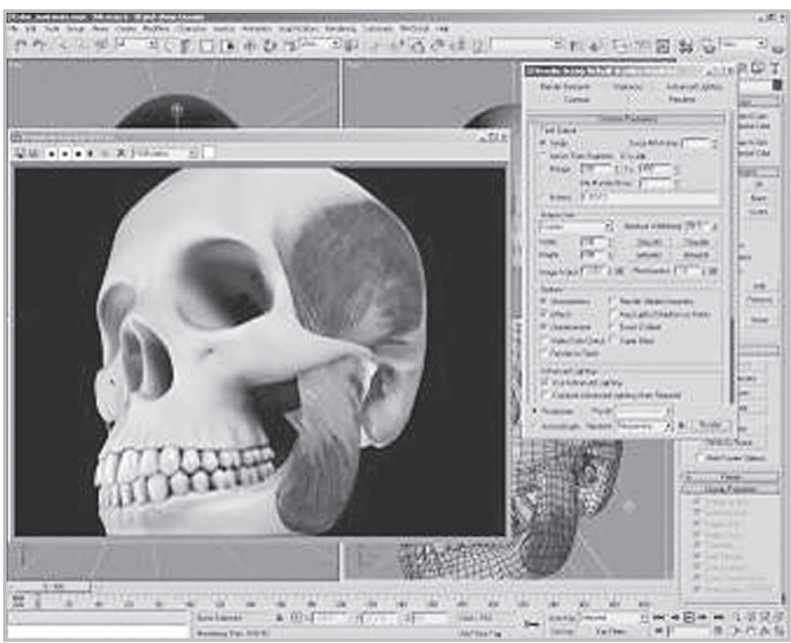

Figura 9 - Renderização. 
- editor HTML;

- programa Dreanweaver;

- programa Front page;

- software para edição de imagem;

- computador pentiun IV e

- 512 megas de memória RAM.

\section{CONSIDERAÇÕES FINAIS}

Este recurso educacional, como objeto de aprendizagem, proporciona a integração dos conhecimentos, desperta a curiosidade e aumenta a velocidade do aprendizado fazendo parte de uma estrutura cognitiva moderna, onde se particicipa de forma consciente como sujeito do processo ensinoaprendizagem.

\section{ABSTRACT}

\section{Development of modern learning objects in Teledentistry}

The purpose of this paper was to show Virtual Man as a learning object and to relate its preparatory stages. Based on the consideration that iconographies must be updated and knowledge must be spread, tools were developed to allow information to be transmitted more efficiently and interactively. Images of high visual and didactic quality were developed by the Virtual Man Project of the Telemedicine Discipline FMUSP (School of Medicine - São Paulo University) together with FOUSP (Dental School - São Paulo University). This learning object makes it possible to watch 3D anatomic structures with physiological movements, observing biomechanics and functional dynamics. This would be impossible to do using conventional methods. The possibility of establishing anatomic correlations, of using transparency resources, subtraction (exclusion) and inclusion of functional dynamics, makes this method a totally new iconography for transmitting large amounts of information in a short period of time, thus improving educational efficiency. The images are used to support student learning and professional training at any dental education institute. This project offers the great benefit of transmitting the knowledge given in hours of theoretical class within only minutes of guided study, thus representing an innovative and a motivating tool. This educational resource is a learning object that integrates knowledge, arouses curiosity and speeds up learning, making it part of a modern cognitive structure, where the individual participates consciously as a subject of the process.

\section{DESCRIPTORS}

Telemedicine. Education, dental. Education, distance. Anatomy.

\section{REFERÊNCIAS}

1. Alencar CJF, Sequeira É, Chao LW, Haddad AE. Soluções de Telemedicina aplicados na Odontologia - Teleodontologia. http://www.ciosp.com.br/anais/Paineis/PalnelEducacao. pdf pg.9-10

2. Alencar CJF, Sequeira É, Chao LW, Haddad AE. Teleodontologia - homem virtual como objeto de aprendizagem - técnica de exodontia de primeiros molares decíduos inferiores. Rev. ABENO, 2007; 7(2):151-152.

3. Bastos LM, Macedo MM. Novas fronteiras da educação: educação à distância. Rev. Bras. Odontol., 2002; 59(2):112-115.

4. Bettio RW, Martins A. Objetos de Aprendizado: um novo modelo direcionado ao ensino a distância. http:/ / www.abed.org. br/congresso2002/trabalhos/texto42.htm

5. Carvalho ACP, Kriger L. Educação Odontológica. Artes Médicas, 2006.

6. Côrrea L. Análise da mudança de paradigma do ensino de graduação em patologia na odontologia: proposta de ensinoaprendizagem a distância via internet. Tese de doutorado apresentada a FOUSP. São Paulo, 2001. 185p.

7. Côrrea L, de Campos AC, Souza SC, Novelli MD. Teaching oral surgery to undergraduate students: a pilot study using a Webbased practical course. Eur. J. Dent. Educ.; 2003; 7(3):111-115.

8. Cunha FS, Silva AE, Larentis NL, Fontanella VRC, Nevado RA. Proposta de uma nova abordagem pedagógica para disciplina de informática aplicada à odontologia. Rev. ABENO, 2005; 5(2):102-108.

9. Chao LW, Silveira PSP, Böhm GM. Telemedicine and Education in Brasil. Journal of Telemedicine and Telecare, 1999; 5:137138.

10. Chao LW, Silveira PSP, Azevedo Neto RS, Böhm GM. Internet discussion lists as an educational tool. Journal of Telemedicine and Telecare, 2000; 6:302-304.

11. Chao LW. Modelo de ambulatório virtual (Cyberambulatório) e tutor eletrônico (Cybertutor) para aplicação na interconsulta médica, e educação à distância mediada por tecnologia.. [tese - Livre Docência] apresentada à Faculdade de Medicina, Universidade de São Paulo; São Paulo; 2003.

12. Chao LW, Enokihara MY, Silveira PSP, Gomes SR, Böhm GM. Telemedicine model for training non-medical persons in the early recognation of melanoma. Journal of Telemedicine and Telecare, 2003;9:S1:4-7.

13. Chao LW, Cestari TF, Bakos L, Oliveira MR, Miot HA, Böhm GM. Evaluation of na Internet-based tedermatology system.. Journal of Telemedicine and Telecare, 2003; 9:S1:9-12.

14. Chao LW. Telemedicina e Telessaúde. Atualidades Brasileiras em Telemedicina \& Telessaúde, 2006; 2(1): 3-5. 
15. Finamor LPS et al. Teleoftalmologia como auxílio diagnóstico nas doenças infecciosas e inflamatórias oculares. Rev. Assoc. Med. Bras., 2005: 51(5):279-284.

16. Haddad AE, Campos FE, Alkmin MB, Chao LW, Rocshke S. Brazilian National Telehealth Program. The International Educational and Networking Forum for e-health, Telemedicine and Health ICT. Proceedings. 2007; p.321-323.

17. Maceratini R, Sabbatini RME. Telemedicina: a nova revolução. Informédica, 1994; 1(6):5-9.

18. Machado LL, Silva JT. Objeto de aprendizagem digital para auxiliary o processo de ensino-aprendizagem no Ensino Técnico em Informática. Novas Tecnologias na Educação CINTED-UFRGS, 2005; 3(2);1-16. http://www.cinted.ufrgs.br/renote/

nov2005/artigosrenote/a23_objeto_aprendizagem_ensinotecnico.pdf

19. Malmström MFV, Marchi MM, Chao LW, Böhm GM. Use of a virtual 3D model for educational purposes em odontology. The $9^{\text {Th }}$ European Congress of DentoMaxilloFacial Radiology, 17-19 of June 2004, Malmö, Sweden, page 33.

20. Malmström MFV, Marta SN, Böhm GM, Chao LW. Homem Virtual: modelo anatômico 3D dinâmico aplicado para educação em odontologia. Revista da ABENO (Associação Brasileira de Ensino Odontológico), Belo Horizonte, 2004; 4(1):87.

21. Massad E, Böhm GM, Chao LW, Silveira PSP. O universo da informática e o ensino médico. Educação Médica. Savier Editora de Livros Médicos Ltda, 211-222, 1998.

22. Oliveira MR, Chao LW, Festa Neto C, Silveira PSP, Rivitti EA, Böhm GM. Web Site for Training Nonmedical Health-Care Workers to Identify Potentially Malignant Skin Lesions and for Teledermatology. Telemedicine Journal and e-Health, 2002; $8(3): 323-332$.

23. Oficina de Telemedicina e Telessaúde - Atenção Primária é foco de projeto de Telemática e Telemedicina. Atualidades Brasileiras em Telemedicina \& Telessaúde, 2006; 2(1):12.

24. Rossi F, Andreazzi D, Chao LW. Development of a Web site for Clinical Microbiology in Brazil. Journal of Telemedicine and Telecare, 2002; 8(S2):14-17.

25. Veronezi MC, Sgavioli CAPP, Böhm GM, Wen LW. Cybertutor: educação mediada por tecnologia na odontologia. Revista da ABENO (Associação Brasileira de Ensino Odontológico), Belo Horizonte, 2004; 4(1):88.

Recebido em 14/10/2010

Aceito em 17/12/2010 